LA CONSTRUCCIÓN DE CIUDADANÍA EN LA ESCUELA SECUNDARIA: CONVIVENCIA, REGULACIÓN DE LA PARTICIPACIÓN JUVENIL y nuevas dinámicas de desigualdad. PÁginas 35-52 en Revista de la Escuela de Ciencias de la Educación, año 10, NÚMERO 9, ENERO A DICIEMBRE DE 2014. ISSN 1851-6297. ISSN EN LINEA 2362-3349.

\title{
LA CONSTRUCCIÓN DE CIUDADANÍA EN LA ESCUELA SECUNDARIA: CONVIVENCIA, REGULACIÓN DE LA PARTICIPACIÓN JUVENIL Y NUEVAS DINÁMICAS DE DESIGUALDAD
}

\author{
Por Pedro Núñez (Universidad de Buenos Aires - CONICET-FLACSO)*, Argentina. \\ pnunez@flacso.org.ar
}

Recibido: 30/06/2014. Aceptado: 15/09/2014

\section{Resumen}

Este trabajo busca problematizar en torno a las formas de construcción de la ciudadanía en la escuela secundaria. Para abordar esta temática la presentación está organizada en tres apartados. En un primer momento, se presenta una breve caracterización de la situación del nivel medio donde se hace referencia a las tasas de cobertura, se describe el proceso de fragmentación educativa y se presentan las distintas tensiones que enfrenta la escuela secundaria en la actualidad.

A continuación, a partir de avances preliminares de una investigación desarrollada en cuatro instituciones ubicadas en la Provincia de Buenos Aires nos preguntamos por la expansión del nuevo sistema de convivencia escolar. Para ello se describen y analizan diferentes "situaciones" o escenas ocurridas en las escuelas con la intención de prestar atención a las formas de regulación de la participación estudiantil, particularmente a las cuestiones que se especifican y las referencias a los derechos de los y las jóvenes. Para ello prestamos atención a tres aspectos: las tradiciones políticas existentes en las instituciones, los grados de heterogeneidad/homogeneidad de la matrícula escolar y los repertorios de acción a los que apelan los y las estudiantes; indagando en las diferencias entre sectores sociales.

\section{Palabras Clave}

Escuela secundaria - Ciudadanía - Inclusión - Convivencia.

* Lic. en Ciencia Política (UBA), Doctor en Ciencias Sociales (UNGS/IDES) e Investigador Asistente del Consejo Nacional de Investigaciones Científicas y Tecnológicas (CONICET) con sede en el Área Educación de la FLACSO. Docente de grado en la Universidad de Buenos Aires y de Posgrado en el Programa UNGS/IDES. 
Revista de la Escuela de Ciencias de la Edducación, año 10, número 9, enero a diciembre de 2014. PÁginas 35-52. ISSN 1851-6297. ISSN EN LINEA 2362-3349. LA CONSTRUCCIÓN DE CIUDADANÍA EN LA ESCUELA SECUNDARIA: CONVIVENCIA, REGULACIÓN DE LA PARTICIPACIÓN JUVENIL Y NUEVAS DINÁMICAS DE DESIGUALDAD. PEDRO NúÑEZ.

\begin{abstract}
This paper seeks to problematize the ways around construction of ci the topics of citizenship in secondary school. To address this issue the presentation is organized into three sections. At first, we made a brief characterization of the state of the education system, with references about coverage rates, the process of massificaction, the educational fragmentation process and described various tensions that secondary school is facing.

Then, from preliminary research advances developed in four institutions situated in Buenos Aires Province we asked for the expansion of the new system of school life and connivance. This describes and analyzes different "situations" that occurred in schools with the intention of paying attention to the forms of regulation of student participation, particularly the issues specified and references to the rights of young people. We do pay attention to three aspects: the existing political traditions in institutions, degrees of heterogeneity / homogeneity of enrollment and youth political actions through an examination of the differences between social classes.
\end{abstract}

\title{
Key Words
}

Secondary school - Citizenship - Inclusion - Connivance.

\section{La expansión de la matrícula del nivel secundario y su impacto en la sociabilidad juvenil}

Hacia inicios de los años sesenta el mundo se sacudía ante la aparición de una nueva "autonomía de la juventud" como estrato social independiente (Hobsbawm, 2003) que dio lugar al surgimiento de una cultura específicamente juvenil. Su advenimiento provocó también la implosión de las categorías con las cuales las ciencias sociales analizaban los fenómenos contemporáneos. El concepto de culturas juveniles rivalizó con los abordajes que caracterizaban a la juventud como aquella etapa de la vida marcada por lo que ya no se es y lo que aún no se ha logrado ser. De forma paralela, el proyecto meritocrático de la escuela moderna comenzó a tambalear con el proceso de masificación del nivel secundario, que ocurrió lenta pero inexorablemente desde la mitad del siglo XX. En los países donde los niveles de cobertura aumentaron más rápidamente -como es el caso de los Estados Unidos, aunque con notorias diferencias raciales- las escuelas secundarias se convirtieron para una gran mayoría de jóvenes en el centro de la vida social, no sólo porque expresaban la posibilidad de acceso a la cultura académica sino también por el hecho de transformarse en un espacio de sociabilidad juvenil. Tal como mostraron diversos estudios, la escuela media se constituyó en el espacio en el cual los y las jóvenes se relacionaban la mayor parte del tiempo entre ellos, aún cuando compartieran el mismo espacio con los adultos (Passerini, 1996). La escuela creaba un mundo con una lógica propia que generaba una ciudad dentro de la ciudad al uso exclusivo de la generación joven (Feixa, 1999). Por otra parte, el 
Revista de la Escuela de Ciencias de la Educación, año 10, número 9, enero a diciembre de 2014. Páginas 35-52. ISSN 1851-6297. ISSN EN LÍNEA 2362-3349. LA CONSTRUCCIÓN DE CIUDADANÍA EN LA ESCUELA SECUNDARIA: CONVIVENCIA, REGULACIÓN DE LA PARTICIPACIÓN JUVENIL Y NUEVAS DINÁmICAS dE DESIGUALdAd. PEdRo NúÑez.

hecho de que los jóvenes pasaran la mayor parte de su tiempo con miembros del propio grupo de edad favorecía el surgimiento de una sociedad adolescente con sus propios lenguajes, símbolos y, más importante todavía, un sistema de valores diferente al establecido en la sociedad más amplia (Coleman citado por Feixa, 1999).

En el caso argentino los procesos cobraron otra temporalidad. Fue a partir de la década del '50 cuando se incorporaron al nivel secundario jóvenes provenientes de las clases populares a partir de la creación de las escuelas técnicas. Los indicadores sociales contribuyen a que podamos darnos una idea de la situación por aquellos años. Si consideramos el nivel de instrucción, de acuerdo a los datos que presentaba Braslavsky (1986) en 1960, alrededor de un $29 \%$ de los jóvenes de 15 a 19 años cuenta con el secundario, pasando en 1970 a alrededor de un 34\%, llegando a casi un 50\% en 1980. Si observamos el siguiente grupo etario que va de los 20 a los 24 años, vemos que en 1960, alrededor de un $20 \%$ tenía nivel secundario, en 1970 , alrededor de un $27 \%$, y en 1980 la cifra asciende a 35\% (Braslavsky, 1986). En nuestro país recién hacia los años '90 la tasa de acceso al nivel secundario supera el $50 \%$ de quienes están en edad de asistir (Southwell, 2011).

La sanción de una nueva Ley Nacional de Educación, ( $\left.N^{\circ} 26.206\right)$, que buscó reorganizar el sistema y poner fin a las crecientes desigualdades entre las jurisdicciones aparece como un nuevo hito, aunque es posible encontrar unos años antes cierta preocupación por la situación de la escuela secundaria, en particular por los diálogos entre la matriz tradicional del nivel y las actuales formas de ser joven. En los diagnósticos pareciera predominar una mirada que enfatiza en la "pérdida" antes que en el surgimiento de innovaciones en su formato y en sus funciones. Mientras algunos análisis se ocuparon de destacar que estamos ante una institución con contenidos perimidos e incapacitada para formar de acuerdo a los actuales requisitos del mundo laboral, muchos otros -con amplia resonancia en los medios de comunicación- refirieron a la preocupación por la convivencia en las aulas, los niveles de violencia entre los alumnos y de ellos hacia sus profesores, y los problemas de aprendizaje, como elementos de fenómenos que "antes no pasaban".

Este escenario fue analizado por diversas producciones académicas que, en esta ocasión, resumiremos en dos grandes líneas de investigación. Por un lado, un conjunto de estudios mostraron que la expansión del nivel se caracterizó por abarcar de manera desigual a la población de acuerdo a la clase social, situación que reavivó las discusiones entre universalismo y particularismo, la unidad del sistema educativo -en particular acerca de su posterior fragmentación- y sobre el ingreso en el espacio escolar de "nuevos fenómenos" como la violencia (Tenti Fanfani, 2003; Tiramonti, 2004; Tiramonti y Ziegler, 2008; Southwell, 2006; Kaplan, 2009). Por otro lado, se encuentran trabajos que hicieron hincapié en los cambios en los sentidos que los jóvenes 
Revista de la Escuela de Ciencias de la Educación, año 10, número 9, enero a diciembre de 2014. Páginas 35-52. ISSN 1851-6297. ISSN EN LINEA 2362-3349. LA CONSTRUCCIÓN DE CIUDADANIA EN LA ESCUELA SECUNDARIA: CONVIVENCIA, REGULACIÓN DE LA PARTICIPACIÓN JUVENIL Y NUEVAS DINÁMICAS DE DESIGUALDAD. PEDRO NúÑEZ.

otorgan a su experiencia educativa (Auyero, 1993; Tenti Fanfani, 2000; Kessler, 2002; Falconi, 2004; Dussel, Brito y Núñez, 2007).

El análisis de las dimensiones más clásicas de la desigualdad (ingreso, calidad de la ocupación, consumo) documenta en el nivel macro las transformaciones sociales ocurridas en el país aunque quizá sea necesario efectuar un estudio más detallado para poder comprender con mayor precisión las implicancias y particularidades de dicho proceso. Una de las dimensiones en las cuales los cambios adquieren mayor visibilidad es la vinculada a las formas que adquiere la sociabilidad. Su análisis permite observar que cada sector social recorre instancias de socialización que privilegian el encuentro con otros similares, configurándose de este modo espacios sociales distantes. Una de las consecuencias que tienen dichos modos de agrupamiento y segregación es que las experiencias de una misma generación poseen pocos elementos en común. El correlato en el mundo educativo de este proceso (que incrementó las distancias sociales) fue la disposición de fragmentos dentro del sistema. Así como la década del ochenta fue terreno fértil para señalar la desigual distribución de oportunidades que tenía lugar al interior del sistema educativo -proceso que configuraba distintos circuitos escolares al diferenciar entre escuelas de mejor y peor calidad y de acuerdo a las modalidades de enseñanza- una década después la preocupación se orientó a la pregunta por las características de la experiencia educativa de los jóvenes, en particular acerca de sus percepciones respecto de los sentidos de ésta en un contexto de desestructuración de los marcos de integración social. Las investigaciones aportaron conocimientos novedosos sobre las experiencias juveniles en un ámbito que gustaba de pensarse igualitario y homogéneo.

El nuevo contexto social puso en cuestión el mito que había contribuido a solidificar la confianza que distintos sectores depositaban en la educación como garante de la igualdad de oportunidades y de las posibilidades de ascenso social. La investigación educativa en el país y la región cuenta con una extensa tradición, desde los años ochenta, en el estudio de la incidencia de los procesos educativos en la producción y reproducción de las desigualdades sociales y educativas. Entre las diversas cuestiones que cobraron preponderancia se destaca la preocupación por lo que ocurre en la escuela secundaria, en particular por los diálogos entre la matriz tradicional del nivel y las actuales formas de ser joven. La progresiva masificación de la escuela media, junto con su reciente condición de obligatoriedad así como la sanción de normativas que reconocen derechos de los y las jóvenes presentan nuevos desafíos no sólo para aquellos encargados de diseñar las políticas públicas del sector, sino para los académicos y los actores que cotidianamente transitan por las escuelas secundarias.

Las experiencias juveniles en la escuela secundaria tienen lugar en un escenario que combina el aumento de la cobertura del nivel secundario con 
Revista de la Escuela de Ciencias de la Educación, año 10, número 9, enero a diciembre de 2014. Páginas 35-52. ISSN 1851-6297. ISSN EN LÍNEA 2362-3349. LA CONSTRUCCIÓN DE CIUDADANÍA EN LA ESCUELA SECUNDARIA: CONVIVENCIA, REGULACIÓN DE LA PARTICIPACIÓN JUVENIL Y NUEVAS DINÁmICAS dE DESIGUALdAd. PEdRo NúÑez.

un sistema educativo caracterizado por su fragmentación, por lo que muchos jóvenes atraviesan una experiencia educativa de baja intensidad (Kessler, 2002). Como consecuencia de este proceso, la socialización entre "nos" reemplaza a la socialización entre diferentes dando lugar a "una socialización entre los iguales, social y culturalmente" (Tiramonti, 2004:13). Si observamos los datos de cobertura es posible apreciar que atravesamos un proceso de creciente masificación del nivel secundario, lo que podría provocar un democratización del espacio -al menos en lo referido a las posibilidades de acceder al mismo por parte de sectores que históricamente estaban excluidos-. Investigaciones recientes señalan que las tasas de cobertura del nivel aumentaron sostenidamente hasta alcanzar para el 2010 en el total del país un promedio de $84,5 \%$ de la población en la franja etaria 13-17 (Montes, 2011). Si hacemos foco en la situación en la Provincia de Buenos Aires hallamos que en dicha jurisdicción se produjo un aumento de la tasa de escolarización del nivel inicial (pasando de 64,3\% en 2001 a 98,0\% en 2010); que se incrementa mínimamente la matrícula del nivel primario — que ya se encontraba casi en el 100\% —; pero que la escuela secundaria preserva los mismos valores luego de diez años (91,3\% en ambos años). En este sentido los datos permiten observar que la sanción de la Ley de Educación Nacional que establece la obligatoriedad del nivel-siguiendo la tendencia de los países del Cono Sur y de México-, antes que un hito en si mismo es un eslabón más de un proceso tendiente a la universalización de la escuela secundaria. Esta suerte de amasetamiento en la cobertura de la escuela media obliga a preguntarse por las causas que dificultan o impiden sostener la escolarización de los jóvenes de estas edades. En este escenario, una de las causas que se destacan es la tensión inherente al vínculo intergeneracional entre "adultos y jóvenes" que asoma como un hecho que, si bien no es novedoso, pareciera extenderse con mayor fricción en la actualidad y que muestra una distancia entre la cultura juvenil y la cultura escolar. En tanto entendemos a las prácticas juveniles como producto de procesos relacionales, antes que consecuencia de las características intrínsecas a "la juventud", consideramos que el estudio aquí propuesto puede brindar pistas fecundas para comprender las características que asumen las relaciones inter e intra-generacionales en la contemporaneidad y sobre cómo facilitan o dificultan las posibilidades de integración al nivel secundario.

Las investigaciones en educación constataron recientemente una correlación entre fragmentación social y fragmentación educativa que conduce a la diversidad de itinerarios y de experiencias escolares de acuerdo a si uno estudia en escuelas donde suelen concurrir las clases medias y altas, en instituciones más tradicionales o surgidas a partir de los años noventa o, incluso, de acuerdo a los distintos formatos escolares. En esta línea, Guillermina Tiramonti (2009) plantea la hipótesis de la incorporación diferenciada de los sectores emergentes 
Revista de la Escuela de Ciencias de la Educación, año 10, número 9, enero a diciembre de 2014. Páginas 35-52. ISSN 1851-6297. ISSN EN LINEA 2362-3349. LA CONSTRUCCIÓN DE CIUDADANIA EN LA ESCUELA SECUNDARIA: CONVIVENCIA, REGULACIÓN DE LA PARTICIPACIÓN JUVENIL Y NUEVAS DINÁMICAS DE DESIGUALDAD. PEDRO NúÑEZ.

en diferentes modalidades y formatos que ponen en tensión las representaciones sobre la igualdad, homogeneidad y diversidad.

La heterogeneidad u homogeneidad de la matrícula se convirtió en una variable determinante. No se trata sólo de una diversidad de trayectorias escolares, hecho constatable en otros momentos históricos, sino del hecho que estas experiencias remiten a universos culturales diferentes, como si no tuvieran relación entre sí o no formaran parte de una misma comunidad. La fragmentación escolar y la construcción de recorridos educativos distintos generó mecanismos de "segregación autoimpuesta" entre sectores de clase media que buscan escenarios de socialización más homogéneos que se manifiestan en la búsqueda de la construcción de espacios de sociabilidad y de una socialización educativa que sea a imagen y semejanza de la socialización familiar.

Son otras generaciones transitando la experiencia por escuelas que no han cambiado tanto. Este punto nos lleva considerar como plausible la hipótesis de la existencia de un proceso de dislocación o desacople (Núñez, 2013) entre la propuesta de la escuela secundaria y las actuales formas de ser joven, más notable aún en aquellos que provienen de sectores populares, quienes son los primeros de su familia en establecer el contacto con la matriz tradicional de la escuela secundaria, pero también constatable de manera transversal en las distintas clases. Esta disociación se manifiesta de múltiples maneras, aunque quizá se plasme de modo más claro en dos aspectos: las disputas por los significados sobre las "funciones de la escuela" y los distintos modos de vivir la "temporalidad". En la actualidad, jóvenes y adultos proyectan esperanzas diferentes sobre lo que debiera ocurrir en el espacio escolar. En cuanto a este primer punto tal vez sea preciso reconocer que dista de ser novedoso, aunque tal vez en la actualidad la divergencia de sentidos se exprese con mayor virulencia. En la escuela secundaria existen entre jóvenes y adultos percepciones discordantes entre sí acerca de lo considerado valioso como saber digno de ser transmitido, distintos usos y valoraciones de las netbooks, celulares y otros dispositivos móviles, sensaciones disímiles acerca de la justicia de las reglas escolares, significados divergentes sobre el modo de entender al respeto o de interpretar diferentes momentos de la vida escolar; cuestiones todas que profundizan la distancia entre docentes/cuerpo directivo y alumnos. Las divergencias, la desconfianza e incluso el desconcierto sobre los modos de participar en la vida en común se expresan de una manera tal que permiten señalar la existencia de una disyunción de corte generacional. (Núñez, 2013). A fin de abordar estas cuestiones en el artículo presentaremos hallazgos de la investigación basada en el Proyecto PIP Vínculos inter e intra generacio- 
Revista de la Escuela de Ciencias de la Educación, año 10, número 9, enero a diciembre de 2014. Páginas 35-52. ISSN 1851-6297. ISSN EN LÍNEA 2362-3349. LA CONSTRUCCIÓN DE CIUDADANÍA EN LA ESCUELA SECUNDARIA: CONVIVENCIA, REGULACIÓN DE LA PARTICIPACIÓN JUVENIL Y NUEVAS DINÁMICAS DE DESIGUALDAD. PEDRO NúÑ̃EZ.

nales en la escuela media: cambios y continuidades en el formato escolar, la convivencia y la construcción de la ciudadanía (1) iniciada en el año 2012 que se desarrolló entre equipos del Área Educación de la Flacso Argentina y de la Universidad Pedagógica Provincial, con financiamiento de esta última institución para problematizar en cuanto a las implicancias que estos procesos tienen en la construcción de la ciudadanía. La muestra estuvo compuesta por dos escuelas tradicionales, de cierta antigüedad (una del interior de la Provincia y otra situada en una localidad de la zona norte del Gran Buenos Aires), como lo son los ex Colegios nacionales de Saladillo (SA) y San Isidro (SI). La particularidad de esta última institución, fundada en 1916, es que históricamente recibió a jóvenes provenientes de los sectores medios y, hasta 2009, tuvo en vigencia un examen de ingreso que le otorgaba un particular perfil de estudiantes. Por su parte, la institución de Saladillo era una escuela nacional transferida a la provincia durante la década de los noventa, hecho al que algunos actores institucionales vinculan a un cierto decaimiento del prestigio y nivel académico del colegio y se relaciona con un cambio en la conformación de la matricula respecto de su nivel socioeconómico. La tercera escuela seleccionada es de modalidad técnica y se encuentra en Campana (CA), localidad de perfil industrial situada a $70 \mathrm{~km}$ de la Ciudad de Buenos Aires. Finalmente, la escuela ubicada en González Catán (GC), parte populoso municipio de La Matanza es una institución surgida en los años '90 a partir de la expansión del sistema educativo. En todas las escuelas se aplicó el mismo modelo de encuesta a una división del anteúltimo año del nivel secundario (quinto año en tres de las escuelas consideradas, cuarto en una), se realizaron entrevistas con directivos, dos docentes, cuatro estudiantes y un preceptor y observaciones del cotidiano escolar.

\section{Sobre las regulaciones de las formas de participación juvenil en la escuela}

En la última década, en muchas ocasiones de forma paralela llevando a la superposición, organismos nacionales y provinciales promovieron un conjunto de leyes y políticas públicas que enuncian los derechos de los y las jóvenes. En ese entramado legislativo institucional se pueden leer las respuestas o iniciativas del Estado en función de determinadas demandas sociales y/o decisiones sobre el direccionamiento de las políticas. La creación del Observatorio Nacional de Violencia Escolar, el Programa de Mediación y Convivencia Escolar, la implementación en algunas jurisdicciones de iniciativas tales como "Jóvenes y Memoria" -en la provincia de Buenos Aires-, y/o la elaboración de nuevas materias como "Construcción de Ciudadanía -, sumado al incentivo en los úl- 
Revista de la Escuela de Ciencias de la Educación, año 10, número 9, enero a diciembre de 2014. Páginas 35-52. ISSN 1851-6297. ISSN EN LINEA 2362-3349. LA CONSTRUCCIÓN DE CIUDADANIA EN LA ESCUELA SECUNDARIA: CONVIVENCIA, REGULACIÓN DE LA PARTICIPACIÓN JUVENIL Y NUEVAS DINÁMICAS DE DESIGUALDAD. PEDRO NúÑEZ.

timos años a la creación y/o consolidación de los Centros de Estudiantes, nos permiten hablar de un repertorio de políticas, a veces no tan coordinado, que busca de diversas maneras una incidencia intencional sobre lo que sucede en el espacio escolar en torno a la ciudadanía (Fuentes y Núñez, 2013).

Uno de los aspectos donde encontramos mayores cambios en las experiencias educativas de las nuevas generaciones refiere a la regulación de las interacciones escolares. Sin lugar a dudas, el impulso de nuevas políticas educativas orientadas a modificar el modo en el que se concebía la "disciplina" en la escuela secundaria representa un escenario novedoso donde se entretejen relaciones basadas en formas de vinculación entre jóvenes y adultos muy diferentes a las que se propiciaban unos años atrás. Por supuesto que estos cambios no impactan de modo similar en todas las instituciones, por lo que podemos señalar que las transformaciones normativas propuestas se caracterizan más bien por su ambigüedad. Aún así, es posible sostener que desde fines del siglo $X X$ se produce un giro en la preocupación de las políticas educativas en relación a las cuestiones disciplinarias, a partir de la incorporación de iniciativas que promovieron el enfoque desde la "convivencia" para resolver los conflictos en el ámbito escolar. Muchas de estas propuestas retoman cuestiones ya planteadas en algunas jurisdicciones en relación a la convivencia democrática y la resolución de conflictos a través de "consejos de convivencia" durante los años ochenta. Estas transformaciones normativas se fundamentaron, de manera esquemática, en dos aspectos. Por una parte, en la esperanza de resolver problemas de conflicto y convivencia actuando desde la construcción de acuerdos colectivos que permitieran mejorar los vínculos entre los actores educativos y construir un marco común de justicia. Por otra parte, en la promoción de la construcción de ciudadanía juvenil por vía de la participación. Ambas cuestiones buscaron discutir, en un nuevo contexto cultural, algunos de los núcleos problemáticos que históricamente organizaron la disciplina en la enseñanza media, basada en la aplicación de "normas de conducta" con miras a la manutención del escolar y la regulación de los estudiantes en dicho espacio.

Observemos detenidamente el nuevo escenario. En primer lugar, cabe destacar la sanción de una nueva Ley Nacional de Educación (N²6206/2006) que busca garantizar la obligatoriedad del nivel secundario, las posteriores adecuaciones de las leyes provinciales así como las resoluciones del Consejo Federal de Educación y del Plan Nacional de Educación Obligatoria y Formación Docente que refieren a los Consejos de Convivencia, los Centros de Estudiantes y la consolidación de una cultura democrática en las escuelas. En segundo lugar, a la par de la conformación de un nuevo entramado legislativo, un número considerable de organismos públicos provinciales impulsó la sanción de nuevas normativas para las escuelas, algunas incluso anteriores a la sanción de ley antes mencionada. A pesar de sus diferencias, las reformas promovidas tienen en común que buscan favorecer la participación y el involucramiento político 
Revista de la Escuela de Ciencias de la Educación, año 10, número 9, enero a diciembre de 2014. Páginas 35-52. ISSN 1851-6297. ISSN EN LÍNEA 2362-3349. LA CONSTRUCCIÓN DE CIUDADANÍA EN LA ESCUELA SECUNDARIA: CONVIVENCIA, REGULACIÓN DE LA PARTICIPACIÓN JUVENIL Y NUEVAS DINÁmICAS dE DESIGUALdAd. PEdRo NúÑez.

de los jóvenes así como intentan producir nuevas dinámicas que mejoren la convivencia en las instituciones escolares. Existen iniciativas a nivel nacional, como el Programa Nacional de Mediación Escolar originado en 2003, el Programa Nacional de Convivencia Escolar y el Observatorio de la Violencia Escolar -creados en 2004-, impulsados por el Ministerio de Educación de la Nación. Durante esos años se promovieron también proyectos a nivel provincial, como el Programa de Convivencia Escolar del Ministerio de Educación del Gobierno de la Provincia de Córdoba, el Programa de Prevención y Asistencia de la Violencia Social en el Ámbito Escolar en La Rioja, el Plan Provincial de Escuelas Abiertas "Un lugar de encuentro" de Salta, el Programa de Mediación Escolar vigente en la Ciudad de Buenos Aires el Programa de Facilitación y Promoción de la Convivencia Escolar de la Ciudad de Rosario, el Programa "Ruedas de Convivencia" en Santa Fe o las modificaciones a los regímenes disciplinarios en la Ciudad de Buenos Aires y la Provincia homónima, por nombrar algunos (2). La preocupación por generar una "buena convivencia" al interior de la escuela media intenta dejar de lado una idea restringida que entiende la disciplina básicamente como una conducta posible de ser categorizada como buena o mala, correcta o incorrecta y se intenta incorporar una concepción más amplia que hace referencia a los modos de estar junto con otros y a la manera en la que es transitada esta experiencia (Litichever y Nuñez, 2005).

Ahora bien, tal como fue señalado por diversos autores, no existe una única manera de entender la cuestión de la convivencia. Isabelino Siede (2007) señala que el término refiere a la regulación de las relaciones entre sujetos diferentes sin que esto implique tener que pensar consensos para cada situación en particular porque la regulación de los conflictos no puede resolverse a través de la negociación permanente de las normas y de una búsqueda constante de consenso ante cada situación particular. Por su parte, Fernández Enguita (2008) enfatiza en que la convivencia con el otro requiere reconocerlo como igual a uno mismo y/o como parte de una misma comunidad y que esto último ocurre cuando lo consideramos sometido a las mismas normas y a formas de vida y valores similares, pero sobre todo lo consideramos titular de derechos y sujeto a obligaciones recíprocas. La convivencia implica una cosmovisión acerca de la vida en común en la escuela que excede con creces la intención de pensarla de modo instrumental solo como forma de "atenuar" o "eliminar" los conflictos (Fridman y Núñez, 2014). Así, podemos tomar dos dimensiones de la convivencia escolar que desarrollan Nilia Viscardi y Nicolás Alonso (2013) a partir de una investigación realizada en Uruguay. Mientras que por una parte la convivencia constituye el conjunto de principios que guían las prácticas y definen los vínculos al interior de las escuelas donde "se conforma, allí, una gramática de la convivencia al enunciarse su sistema de reglas y normas" (2013:29).

En uno de los primeros estudios que observaron en detalle las cuestiones que regulaban los Acuerdos de Convivencia Inés Dussel (2005) mostró que la 
Revista de la Escuela de Ciencias de la Educación, año 10, número 9, enero a diciembre de 2014. Páginas 35-52. ISSN 1851-6297. ISSN EN LINEA 2362-3349. LA CONSTRUCCIÓN DE CIUDADANIA EN LA ESCUELA SECUNDARIA: CONVIVENCIA, REGULACIÓN DE LA PARTICIPACIÓN JUVENIL Y NUEVAS DINÁMICAS DE DESIGUALDAD. PEDRO NúÑEZ.

mayoría de los Reglamentos de escuelas secundarias de la Ciudad de Buenos Aires que analizó consideraban a los jóvenes como incapaces y objeto de deberes y obligaciones, pero sujetos con pocos derechos. Estas nuevas normativas regulaban sólo a los alumnos, expresión del predominio en la ley escolar de la preocupación por su indisciplina. Asimismo, de acuerdo a la autora, la ausencia de regulación de los adultos dificultaba las posibilidades de construir un marco común de justicia.

Cuando nos acercamos a conocer las visiones de los actores educativos encontramos que, a pesar de estos cambios, una gran mayoría de docentes y alumnos al ser consultados sobre las normativas vigentes en sus escuelas destaca una descontextualización entre las normas estipuladas en el reglamento y la realidad que enfrentan, situación que obliga a los distintos actores a improvisar estrategias. Las investigaciones que abordaron el estudio de estas cuestiones hallaron que las innovaciones tuvieron un impacto diferencial en las escuelas; los Consejos de Convivencia han funcionado de forma mucho menos regular, pero, por lo general, los Reglamentos se han instalado como un documento legítimo que contiene aquellas normas consensuadas para regular la conducta de los actores educativos; aunque son pocos los casos en los que su elaboración había sido producto de la reflexión conjunta y compartida por los distintos actores (Litichever y Nuñez, 2011).

Encontramos que la mayoría del articulado de las leyes o resoluciones de convivencia escolar continuaban pensando en la convivencia como sinónimo de sanciones escolares (Fridman, 2013; Litichever, 2012) aunque se incluyeron cambios en los modos de armado de las normas y de aplicación de las sanciones. En su tesis de maestría Litichever (2010) demuestra que, a pesar de la constatación de la persistencia de estas formas más tradicionales de organización escolar, en muchos de los Reglamentos de Convivencia de las veinticuatro escuelas en las cuatro jurisdicciones que examinó, hacen referencia a distintas leyes y convenciones sobre las que se apoyan: la Constitución Nacional, Tratados Internacionales, la Declaración Universal de los Derechos Humanos, la Convención sobre los Derechos de niños, niñas y adolescentes, y leyes jurisdiccionales, inscribiendo su marco normativo en un marco legal más amplio que podría llegar a brindar a los estudiantes un conocimiento mayor de los derechos y obligaciones. Uno de los principales hallazgos a los que arriba es la existencia de cierta correlación entre los estilos institucionales de las escuelas y los Reglamentos de Convivencia elaborados por las mismas. Según Litichever, existe una consonancia entre el sector social de la población que la escuela suele atender y los estilos de los reglamentos, lo que produce que varíen notablemente en cada escuela.

¿Cuál es el impacto de estas transformaciones normativas en el espacio escolar? ¿Qué características tienen los nuevos Acuerdos de Convivencia? ¿Qué regulaciones existen sobre las formas de participación política? ¿Qué 
Revista de la Escuela de Ciencias de la Educación, año 10, número 9, enero a diciembre de 2014. Páginas 35-52. ISSN 1851-6297. ISSN EN LÍNEA 2362-3349. LA CONSTRUCCIÓN DE CIUDADANÍA EN LA ESCUELA SECUNDARIA: CONVIVENCIA, REGULACIÓN DE LA PARTICIPACIÓN JUVENIL Y NUEVAS DINÁMICAS DE DESIGUALDAD. PEDRO NúÑ̃EZ.

cuestiones se especifican y en qué documentos? En las páginas que siguen presentamos algunos ejemplos con la intención de problematizar sobre estas cuestiones.

\section{a) Las regulaciones en los Reglamentos de Convivencia}

Una manera de operacionalizar el estudio de los modos de hacer "política" en las instituciones es indagar en la regulación de su práctica, es decir, si los documentos y normativas promueven la organización estudiantil, si los Acuerdos de Convivencia incorporan temáticas acerca de los derechos de los jóvenes y si existen menciones específicas. A pesar de la referencia normativa común, por el hecho de ser parte de la misma jurisdicción, la situación en las cuatro escuelas consideradas en nuestra investigación es marcadamente diferente. En la investigación nos encontramos con una traza particular que posiblemente remita a las tradiciones existentes en las distintas modalidades del nivel medio. Las dos instituciones que contaban con Centro de Estudiantes eran los ex Colegios Nacionales. En el caso de la Escuela Técnica en Campana un grupo de jóvenes quiso organizarlo, pero se encontró con no pocas dificultades, tanto para concentrar el interés de otros compañeros como por la falta de apoyo de algunos directivos. Finalmente, en la Escuela de González Catán no funcionaba este tipo de instituciones, aunque sí había delegados por curso.

Por otra parte, las cuatro instituciones contaban con un documento que podría reconocerse como Acuerdo de Convivencia, aunque en el caso de Saladillo era donde mayor extensión y abordaje de situaciones plantea. El Reglamento de SI se enfocaba a la tipificación de normas e instituciones a regular, el de La Matanza combinaba intentos de incorporar nuevos aspectos en relación a la convivencia y la persistencia de regulaciones tradicionales mientras que el de Campana se trataba más bien de un Régimen de asistencia y puntualidad de los alumnos. Como es factible apreciar, existe una disparidad de situaciones en relación a Centros de Estudiantes y Acuerdos de Convivencia que establecen pisos distintos de referencias de derechos para las y los jóvenes. Sin contar con argumentos suficientes para señalar que esta situación implica relaciones desiguales, es posible sostener que dicha disparidad implica diferencias en lo que Fraser denomina "paridad en la participación" (2011) y que nos encontramos ante situaciones en las que las diferencias, en caso de ser consideradas injustas por las y los jóvenes, podrían convertirse en desigualdades (Therborn, 2004).

En los Reglamentos consultados encontramos algunas referencias a la participación política, y aquí es factible señalar diferencias entre las instituciones. Las dos escuelas ex nacionales (San Isidro y Saladillo) mencionan de manera genérica la participación de los estudiantes mientras que en González Catán, si bien aparece una enunciación similar, también es el único que incorpora en el documento una sanción específica. Este Acuerdo, al igual que otros documentos regula el horario de entrada al establecimiento y plantea situaciones especiales 
Revista de la Escuela de Ciencias de la Educación, año 10, número 9, enero a diciembre de 2014. Páginas 35-52. ISSN 1851-6297. ISSN EN LINEA 2362-3349. LA CONSTRUCCIÓN DE CIUDADANIA EN LA ESCUELA SECUNDARIA: CONVIVENCIA, REGULACIÓN DE LA PARTICIPACIÓN JUVENIL Y NUEVAS DINÁMICAS DE DESIGUALDAD. PEDRO NúÑEZ.

por trabajo, embarazo y maternidad (aunque nada dice sobre la paternidad); la circulación por la escuela en horarios de clase y recreos; la vestimenta e incorpora en el contenido del Reglamento la sanción de las agresiones y la discriminación así como busca preservar el cuidado del material de la institución.

Un punto llamativo, directamente vinculado a la discusión que planteamos, es el que hace referencia a que "la circulación de panfletos partidarios está terminantemente prohibida dentro de la escuela" (Punto 4). Esta medida pareciera ser manifiestamente contraria a la intención de propiciar la conformación de Centros de Estudiantes en las instituciones y sitúa a la "política" como cuestión que atañe a los adultos. La regulación explícita de las formas de participación política estudiantil no cuenta con el respaldo de una normativa específica ya que ni en el Reglamento General de Instituciones Educativas ni, como observamos, en las Leyes y resoluciones específicas aparece dicha prohibición. A su vez, permite interrogarse sobre las diferencias en cuanto a la circulación de "panfletos", listas, folletos o afiches de los sindicatos docentes, actividad absolutamente legitimada por el derecho a agremiación de los docentes y habitual en las instituciones.

El ejemplo de la Escuela de GC nos habla de la persistencia en el nivel secundario de una definición de la participación política que la piensa desvinculada de los partidos políticos. lara Enrique señala que ya a inicios de los años ochenta, incluso cuando de manera concomitante se promovieron las prácticas participativas de los y las jóvenes, era posible hallar la persistencia de ciertos temores a los vínculos con partidos políticos (Enrique, 2010). Trabajos realizados hacia fines de la década del 2000 dieron cuenta de las disputas que suceden en las instituciones a la par del intento de conformación del Centro de Estudiantes en una escuela de la zona oeste del Gran Buenos Aires (Castro, 2007). Por su parte, Marina Larrondo (2012) en su investigación sobre el movimiento estudiantil secundario en la Provincia, señala que los estudiantes deben "camuflar" sus identificaciones políticas cuando asisten a las escuelas.

En contraste, la Escuela de Saladillo plantea en su Acuerdo Institucional de Convivencia la necesidad de "estar dispuestos a asumir los riesgos de que otro participe en tanto que participar es tomar decisiones" (Pág 1, Presentación) y en la institución se promovió la realización de una Jornada de Convivencia, en coordinación con el Centro de Estudiantes y delegados de curso, para trabajar sobre la redacción del documento. Cuando estuvimos en la escuela se realizaban las elecciones del Centro y pudimos observar que en los carteles de las listas, a la sazón y para evitar vínculos partidarios llamadas simplemente Lista 1 y Lista 2, la organización de dicha Jornada era señalada como un logro de los estudiantes. Finalmente, la Escuela SI también menciona en su Acuerdo como una norma más que como un derecho que se espera que como "integrante" de esa institución propicie "con su participación la creación de un clima adecuado y respetuoso por la tarea educativa" (Punto 5) y la elección de tres 
Revista de la Escuela de Ciencias de la Educación, año 10, número 9, enero a diciembre de 2014. Páginas 35-52. ISSN 1851-6297. ISSN EN LÍNEA 2362-3349. LA CONSTRUCCIÓN DE CIUDADANÍA EN LA ESCUELA SECUNDARIA: CONVIVENCIA, REGULACIÓN DE LA PARTICIPACIÓN JUVENIL Y NUEVAS DINÁMICAS DE DESIGUALDAD. PEDRO NúÑ̃EZ.

alumnos para que participen del Consejo de Aula, espacio del que participan el preceptor, tutor, profesor y los estudiantes para resolver situaciones de conflicto. Tal como puede apreciarse existen matices en la promoción y regulación de la participación política juvenil que proyectan modos diferentes, y podríamos pensar desiguales, de construcción de la ciudadanía.

\section{b) Tensiones por los carteles y los panfletos}

Por razones que toda investigación enfrenta, en este caso para recopilar nuevos materiales, un año después de la primera etapa del trabajo de campo -que coincidió como dijimos con la discusión del proyecto de ley sobre voto joven- debimos regresar a la Escuela SI. Se trata de una escuela que cuenta con una extensa tradición de organización estudiantil, el Centro de Estudiantes organiza el acto del 16 de Septiembre (3) y es parte sustancial del proyecto escolar, reconocido tanto por directivos y docentes como por muchos estudiantes. En la encuesta les consultamos acerca de cuáles eran los aspectos que más les gustan de su escuela. Se trataba de una pregunta de respuesta múltiple, por lo que podían indicar varias cuestiones. En el caso de esta escuela el 30\% de las menciones hizo referencia a "las actividades del Centro de Estudiantes" (que no recogió alusiones en dos escuelas y en Saladillo concentró sólo 6,9\% de menciones) mientras que $43,3 \%$ señalaba "el clima que hay en la escuela" y la más aludida fue "que podemos participar, opinar" (50\%). Como vemos, la referencia concreta al espacio "Centro de Estudiantes" así como la percepción de un clima escolar que posibilita la participación aparece como una dimensión clave de pertenencia institucional e integración.

En la elección de autoridades del Centro de ese año habían participado más de ochocientos estudiantes que optaron entre dos listas "Voz Secundaria" y "Papel secundario". La primera resultó ganadora, con 460 votos y se presenta como "independiente", aunque varios de sus integrantes militan en partidos de izquierda. A pesar de que la elección había transcurrido con absoluta tranquilidad, durante los días del trabajo de campo la escuela atravesaba una situación conflictiva debido a que habían circulado panfletos con referencias políticas y uno de los estudiantes había sido sancionado. Las paredes del establecimiento contaban con carteles que denunciaban la "persecución" a los estudiantes. En la entrevista que realizamos con uno de los integrantes del Centro de Estudiantes señaló que el cartel en cuestión invitando a una marcha convocada por un "partido político" había sido pegado en algunas paredes, razón por la cual los directivos citaron a los padres del alumno identificado como responsable del hecho. De acuerdo al rumor que circulaba, las autoridades les habrían señalado que "la militancia afectaba" el comportamiento del joven.

Los estudiantes habían realizado diversas acciones: la semana previa a nuestra visita a la escuela organizaron una sentada en la puerta del establecimiento y no ingresaron a clases y para el fin de semana siguiente preparaban un 
Revista de la Escuela de Ciencias de la Educación, año 10, número 9, enero a diciembre de 2014. Páginas 35-52. ISSN 1851-6297. ISSN EN LINEA 2362-3349. LA CONSTRUCCIÓN DE CIUDADANIA EN LA ESCUELA SECUNDARIA: CONVIVENCIA, REGULACIÓN DE LA PARTICIPACIÓN JUVENIL Y NUEVAS DINÁMICAS DE DESIGUALDAD. PEDRO NúÑEZ.

festival cultural con la consiga "Basta de persecución política a los estudiantes. Exigimos plena libertad de expresión". De acuerdo a uno de los entrevistados, reclamaban poder difundir sus ideas políticas en la escuela por una razón sencilla: "hay carteles de la provincia, del ministerio de Educación y nosotros no podemos pegar carteles".

Más allá de la citación al alumno y su resolución -e incluso de la discusión sobre las consignas utilizadas como la noción de "persecución política"- nos interesa problematizar en torno las tensiones que enfrenta la escuela secundaria. A modo de hipótesis sostenemos que las escuelas se ven desbordadas ante un nuevo contexto que combina la mayor visibilización de las juventudes en el nivel secundario (incluso de sectores sociales históricamente excluidos del nivel) y nuevas leyes, normativas y programas que fomentan la participación juvenil. En este escenario es sintomático que estudiantes y docentes sostengan que la "política partidaria está prohibida". ¿Cómo repercuten estas cuestiones en la conformación de vínculos intergeneracionales? ¿Qué estrategias llevan adelante los estudiantes y cuáles los docentes?

Es plausible pensar que el intento de diferenciar entre una "buena" y una "mala" política -la primera vinculada a actividades gremiales o culturales, la segunda a lo partidario- es un intento por parte de los docentes y directivos de resituar el límite entre un adentro y un afuera escolar-. Se impone una figura de la ciudadanía como "ensayo", como preparación para la vida adulta y para ejercer en espacios fuera de la escuela. Así como en otros aspectos algunas escuelas enfrentan el proceso de masificación de maneras innovadoras, la política continúa siendo pensada como reducto a preservar en poder de los adultos. Las normativas, así como pueden ser elementos intangibles a los cuales los estudiantes apelen "por sus derechos", establecen modos correctos de participar. A la vez, en toda institución se producen formas legítimas e ilegítimas de participación política que pretenden moldear las prácticas de los sujetos.

Cuando decimos que las instituciones están desbordadas no planteamos un proceso de des-institucionalización sino más bien la manifestación de nuevas líneas de diferenciación al interior del sistema, que presenta formas de construcción de la ciudadanía y de los "modos de hacer política" que asoman como diferentes y desiguales. Implica también sostener que las autoridades educativas en distintos niveles -tanto los más entusiastas promotores de las iniciativas como para quienes las enfrentan subrepticiamente- deben lidiar con formas de participación política juvenil que pueden no ajustarse a los deseos de los adultos, o, al menos, tomar carriles diferentes a los diseñados. Asimismo, el nuevo escenario promueve la aparición de la identificación política de los jóvenes como reafirmación de su lugar en la institución, como una estrategia más en su búsqueda de hacerse notar en el espacio escolar. 
Revista de la Escuela de Ciencias de la Educación, año 10, número 9, enero a diciembre de 2014. Páginas 35-52. ISSN 1851-6297. ISSN EN LÍNEA 2362-3349. LA CONSTRUCCIÓN DE CIUDADANÍA EN LA ESCUELA SECUNDARIA: CONVIVENCIA, REGULACIÓN DE LA PARTICIPACIÓN JUVENIL Y NUEVAS DINÁMICAS DE DESIGUALDAD. PEDRO NúÑ̃EZ.

\section{Apuntes finales: hacia nuevas líneas de investigación}

En este trabajo buscamos presentar ejemplos de situaciones ocurridas en escuelas secundarias en la Provincia de Buenos Aires con la intención de problematizar en torno a las formas de construcción de la ciudadanía en el espacio escolar. Creemos que es posible encontrar algunas diferencias en la "construcción de ciudadanía" que se deben principalmente a los grados de homogeneidad/heterogeneidad de la matrícula de cada establecimiento y a las tradiciones políticas existentes y a las prácticas políticas. La ciudadanía adquiere la fisonomía de distintas "figuras": jóvenes que inscriben sus acciones en las tradiciones políticas existentes -fundamentalmente el Centro de Estudiantes-, otros que apelan a formas novedosas de expresión -en una combinación de expresiones culturales-, pero también quienes buscan "esforzarse", sacrificarse" y que pretenden soluciones concretas a las necesidades que enfrentan cotidianamente en sus escuelas.

De manera esquemática, es posible destacar que los jóvenes de "sectores medios" y altos se quejan sobre las diferencias que hacen las autoridades entre los alumnos (Núñez, 2013). Por su parte, quienes provienen de las clases medias-bajas y populares -en su mayoría primera generación en sus familias en acceder al nivel secundario- resaltan las diferencias en la aplicación de la ley entre alumnos y docentes, haciendo hincapié tanto en que las normas suelen prescribir únicamente las conductas de los estudiantes como, principalmente, en la falta de sanción al ausentismo docente o la carencia en la enseñanza de contenidos escolares - precisamente aquello que diferencia a una escuela de otra institución-. No desconocemos las dificultades en establecer una definición apriorística (Visacovsky Garguin, 2009) que permita considerar qué instituciones cuentan con una matrícula proveniente en su mayoría de las clases medias, pero entendemos que al focalizar en las prácticas y relatos de las y los jóvenes que participan de la delimitación de cada sector social logramos complejizar la mirada en torno a los repertorios de acción política más allá de las definiciones clásicas. Tal como señala Visacovsky (2008), de esta forma la definición se abre a las variables como las imágenes corporales y espaciales, los valores y las aspiraciones y, en esta misma orientación, las formas de construcción de la ciudadanía aparecen como un elemento central en la (auto) percepción de los estudiantes como miembros de determinado sector social así como de los docentes sobre el origen social de sus alumnos lo que imagina o torna inviable ciertos aprendizajes y prácticas políticas.

Las experiencias educativas juveniles adquieren formas disímiles que podrían redundar en nuevas formas de desigualdad. La idea de ciudadanía implica grados de pertenencia y reconocimiento (Jelin, 1997), de integración a un común a partir de la percepción de contar con los mismos derechos, por lo que es preciso problematizar en las percepciones juveniles sobre la noción así como los sentidos que le otorgan en un contexto de fragmentación y segregación 
Revista de la Escuela de Ciencias de la Edducación, año 10, número 9, enero a diciembre de 2014. PÁginas 35-52. ISSN 1851-6297. ISSN EN LINEA 2362-3349. LA CONSTRUCCIÓN DE CIUDADANÍA EN LA ESCUELA SECUNDARIA: CONVIVENCIA, REGULACIÓN DE LA PARTICIPACIÓN JUVENIL Y NUEVAS DINÁMICAS DE DESIGUALDAD. PEDRO NúÑEZ.

socio-educativa. La tendencia a la masificación del nivel secundario supone una apropiación por parte de los jóvenes del espacio escolar. Sin embargo, a este punto de partida desde posiciones iguales - todos tienen acceso - le suceden maneras diferentes de transitar por la escolarización que podrían implicar modos de formación ciudadana disímiles y concepciones sobre la "política", la "igualdad", la "justicia" o los "derechos" diferentes.

\section{Notas Bibliográficas}

(1) El proyecto estuvo dirigido por Myriam Southwell y, además del autor sus integrantes eran Lucía Litichever, Denise Fridman, Jaime Piracón, Fabiana Guzzini, Gabriel Tolosa Chacón, Luisa Vecino, Bárbara Guevara y Virginia Rodriguez. Su objetivo fue el estudio de las formas en las que se estructuran las relaciones inter e intra-generacionales en la institución escolar para repensar temáticas como la convivencia, la ciudadanía o el uso de las TIC. La continuación de esta investigación se está llevando a cabo en el marco del PICTO 2012 UNIPE -ANPCyT Nro. 97 "Escuela media y cultura contemporánea: vínculos generacionales, convivencia y formación ciudadana" bajo la dirección de Myriam Southwell. La investigación se realizó en cuatro instituciones de la Provincia de Buenos Aires

(2) En el primer caso, en 2001 fue aprobado el Sistema Escolar de Convivencia (Ley 223 de 1999, reglamentada por el Decreto $\mathrm{N}^{\circ} 1400 / 001$ de 2001) que deroga la antigua legislación nacional de 1943 (Decreto №150.073). Por su parte, en la Provincia de Buenos Aires entra en vigencia una nueva Resolución en el 2002 (Resolución NN1593/02) que revoca la norma anterior de 1958 (1709/58). La normativa propicia la formación de Acuerdos Institucionales de Convivencia con el propósito de «concretar una convivencia democrática en el nivel polimodal», y la puesta en marcha de Consejos de Convivencia Escolar encargados de regir la convivencia en la escuela.

(3) El 16 de Septiembre de 1976 un grupo de estudiantes secundarios de la Ciudad de La Plata que reclamaban por el "boleto estudiantil" fueron secuestrados por grupos de tareas durante la última dictadura militar. La mayoría continúa desaparecidos. Federico Lorenz (2004) destaca que la conocida como "La Noche de los Lápices" se convirtió en un emblema que cobró una dimensión política sumamente atractiva, al punto que desde los años ochenta existen un sinnúmero de agrupaciones que toman como nombre "16 de Septiembre" y referencias similares que funcionan como homenaje a los jóvenes desaparecidos y ligazón con las experiencias políticas de las generaciones precedentes. Este vínculo se ha consolidado con el tiempo, al punto que en muchas instituciones se realiza un acto, generalmente organizado por los estudiantes y existen marchas conmemorativas en distintas localidades del país (no está de más recordar que en 2006 se instauró por decreto del entonces Presidente Néstor Kirchner, el 16 de Septiembre como Día Nacional de la juventud).

\section{Referencias Bibliográficas}

- $\quad$ Auyero, J. (1993). Otra vez en la vía. Notas e interrogantes sobre la juventud de sectores populares. Buenos Aires: Espacio Editorial.

- Braslavsky, C. (1986). Informe de situación de la juventud argentina. Buenos Aires: CEAL. 
Revista de la Escuela de Ciencias de la Educación, año 10, número 9, enero a diciembre de 2014. Páginas 35-52. ISSN 1851-6297. ISSN EN LINEA 2362-3349. LA CONSTRUCCIÓN DE CIUDADANÍA EN LA ESCUELA SECUNDARIA: CONVIVENCIA,

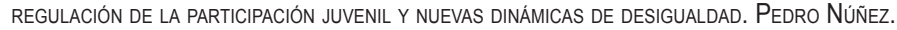

- Dussel, I. (2005). ¿Se renueva el orden disciplinario escolar? Una lectura de los reglamentos de convivencia en la Argentina de la post-crisis". Revista Mexicana de Investigación Educativa, 10 (27).

- Dussel, I., Brito, A. y Nuñez, P. (2007). Más allá de la crisis. Visión de alumnos y profesores de la escuela secundaria. Buenos Aires: Santillana.

- Enrique, I. (2010). El protagonismo de los jóvenes estudiantes en los primeros años de democracia (1983-1989).Actas de II Reunión Nacional de Investigadores/as en Juventudes de Argentina. Octubre, Salta, Argentina.

- $\quad$ Feixa, C. (1999) [1998]. De jóvenes, bandas y tribus. Barcelona: Ariel.

- Fernández Enguita, M. (2008). Escuela y ciudadanía en la era global. En Diker, G. y Frigerio, G. (comps.) Educar: Posiciones acerca de lo común. Buenos Aires: Del Estante.

- Fraser, N. (2010). Injustice at intersecting scales: on social exclusión and the global poor. European Journal of Social Theory, Sage Publications, 13.

- $\quad$ Fridman, D. (2013). Cambios normativos en la regulación de los sistemas de convivencia escolar. CABA: CLACSO, Red CLACSO de posgrados

- $\quad$ Fridman, D. y Núñez, P. (2014). Figuras de ciudadanía en la escuela secundaria: configuraciones normativas y formas de participación política en la Provincia de Buenos Aires, Argentina. En Mayer, L. y Unda, R. (comp.) Procesos y experiencias de socialización escolar en Iberoamérica. CLACSO, Universidad de Manizales. En prensa.

- Fuentes, S. y Núñez, P. (2012). Estudios sobre ciudadanía en la escuela secundaria: un repaso de las perspectivas predominantes en las investigaciones en la última década (2002-2012). Buenos Aires: Mimeo.

- Hobsbawm, Eric (2003) [1994] Historia del siglo XX. Buenos Aires: Crítica.

- Jelin, E. (1997). Ciudadanía de las mujeres en América Latina. Revista Agora. Cuaderno de Estudios Políticos,(7)

- Kaplan, C. (2009). Violencia escolar bajo sospecha. Buenos Aires: Miño y Dávila.

- Kessler, G. (2002) La experiencia educativa fragmentada. Estudiantes y docentes en la escuela media en Buenos Aires. Buenos Aires: IIPE- UNESCO.

- Larrondo, M. (2012) Lápices de colores. El movimiento estudiantil secundario en Argentina: Investigaciones recientes. CLACSO-Red Inju . En prensa.

- Litichever, L. (2010). Los Reglamentos de Convivencia en la Escuela Media. La producción de un orden normativo escolar en un contexto de desigualdad. Tesis de Maestría en Ciencias Sociales con Orientación en Educación. Buenos Aires, FLACSO. Argentina

- $\quad$ Litichever, L. y Núñez, P. (2005) Acerca de lo justo, lo legal y lo legítimo. Cultura política en la escuela media. Revista Última Década, (23). Valparaíso: CIDPA.

- Lorenz, F. (2004). 'Tómala vos, dámela a mí: La noche de los lápices, el deber de memoria y las escuelas. En Jelin, E. y Lorenz, F. (comps.). Educación y memoria: La escuela elabora el pasado. Buenos Aires: Siglo XXI.

- Montes, N. (2011). Principales rasgos de la educación en la región desde la información estadística disponible. Revista Propuesta Educativa, (35).

- Núñez, P. (2013) La política en la escuela. Jóvenes, justicia y derechos en el espacio escolar. Buenos Aires: La Crujía.

- Passerini, L. (1996). La juventud, metáfora del cambio social (dos debates sobre los jóvenes en la Italia fascista y en los Estados Unidos durante los años cincuenta). En Levi, Giovanni y Schmitt, J. C. (comp.)Historia de los jóvenes. Madrid: Taurus.

- Siede, I. (2007) La educación política. Ensayos sobre ética y ciudadanía en la escuela. Buenos Aires: Paidós. 
Revista de la Escuela de Ciencias de la Edducación, año 10, número 9, enero a diciembre de 2014. PÁginas 35-52. ISSN 1851-6297. ISSN EN LÍNEA 2362-3349. LA CONSTRUCCIÓN DE CIUDADANIA EN LA ESCUELA SECUNDARIA: CONVIVENCIA, REGULACIÓN DE LA PARTICIPACIÓN JUVENIL Y NUEVAS DINÁmiCAS dE DESIGUALDAD. PEDRO NúÑez.

- Southwell, M. (2006). La tensión desigualdad y escuela. Breve recorrido histórico de sus avatares en el Río de la Plata. En Martines, Pablo y Redondo, Patricia (comp.) Igualdad y Educación. Escrituras entre (dos) orillas. Buenos Aires: Del Estante.

- Tenti Fanfani, Emilio (2000) Culturas juveniles y cultura escolar. Buenos Aires: IIPE UNESCO.

- Thernborn, G. (2006) Inequalities of the world. Londres: Verso.

- Tiramonti, G. (2009) La escuela media en debate, Buenos Aires: FLACSO-Manantial.

- Tiramonti, G. (dir.) (2011) Variaciones sobre la forma escolar. Límites y posibilidades de la escuela media. Buenos Aires: FLACSO-Homo Sapiens.

- Tiramonti, G. (comp.)(2004) La trama de la desigualdad educativa. Mutaciones recientes en la escuela media. Buenos Aires: Manantial.

- Visacovsky, S. y Garguin, E. (2009). Introducción. En Visacovsky, S. y Garguin, E. (comp.) Moralidades, economías e identidades de clase media. Estudios históricos y etnográficos. Buenos Aires: Ed. Antropofagia.

- Viscardi, N. y Alonso, N. (2013). Gramática (s) de la convivencia. Montevideo: ANEP. 